\title{
La verificación periodística frente a la mentira en los procesos electorales de Ecuador y España ${ }^{1}$
}

\author{
Journalistic verification against \\ lies in the electoral processes of Ecuador and Spain
}

\author{
Claudia Rodríguez-Hidalgo \\ Universidad Técnica Particular de Loja \\ cvrodriguezx@utpl.edu.ec \\ https://orcid.org/0000-0003-4004-9389 \\ Javier Herrero \\ Universidad de Salamanca \\ javiherrero82@usal.es \\ https://orcid.org/0000-0002-1362-7406 \\ Ignacio Aguaded-Gómez \\ Universidad de Huelva \\ aguaded@uhu.es \\ https://orcid.org/0000-0002-0229-1118
}

Forma sugerida de citar: Rodríguez-Hidalgo, C., Herrero, J., \& Aguaded-Gómez, I. (2021). La verificación periodística frente a la mentira en los procesos electorales de Ecuador y España. Universitas, 34, pp. 41-62.

1 Este escrito fue desarrollado durante la estancia de investigación realizada en la Universidad de Salamanca, bajo la tutela del profesor Javier Herrero-Gutiérrez, cofinanciada por la propia institución española dentro del programa de la USAL "Movilidad de profesores extranjeros en el marco de convenios". 


\title{
Resumen
}

La verificación de hechos es clave en el marco de procesos electorales, el discurso político nunca estuvo tan expuesto como hoy al escrutinio público y a la posibilidad de ser desmentido en vivo y de forma masiva. Esta investigación se enfoca en los proyectos de verificación informativa de Ecuador y España, y en su rol en las elecciones de ambos países en marzo y abril de 2019, respectivamente.

Se analizaron las publicaciones más relevantes de ambos proyectos en Twitter con el objetivo de identificar los principales rumores difundidos y sometidos a verificación, así como los políticos sobre los cuáles se difundió la mayor cantidad de rumores.

Los resultados más relevantes muestran que las declaraciones falsas de candidatos y la migración fueron los temas principales de las fake news en ambos países, y que las mentiras en torno a determinados candidatos no afectó de modo determinante los resultados de las elecciones. Del mismo modo se evidenció que existe una marcada desigualdad en la difusión de la información verificada en ambos países, puesto que estas no reflejan la misma intensidad en cuanto a la producción de desmentidos, así como a la difusión de estos.

\section{Palabras clave}

Periodismo, información, internet, comunicación, medios sociales, campaña política.

\begin{abstract}
Fact-checking is key in the framework of electoral processes, political discourse has never been so exposed as it is today to public scrutiny and the possibility of being denied live and in a massive way. This research focuses on the fact-checking projects in Ecuador and Spain, and their role in the elections of both countries in March and April 2019, respectively.

The most relevant publications from both projects on Twitter were analyzed, the aim was to identify the main rumors that were spread and subject to verification, as well as the politicians about whom the most rumors were spread.

The most relevant results it is evident that the immigration issue and false declarations by candidates were the main topics of the fake news in both countries, and that the lies about certain candidates did not decisively affect the results of the elections. Similarly, it was evident that there is a marked inequality in the dissemination of verified information in both countries, since these do not reflect the same intensity in terms of the production of denials, as well as the dissemination of these.
\end{abstract}

\section{Keywords}

Journalism, information, internet, communication, social media, political campaign.

\section{Introducción}

Los rumores y mentiras son comunes en las campañas electorales. El uso de estas estratagemas coadyuva a desdibujar la imagen de un candidato frente a sus electores y se acompaña de prácticas cuestionables que se esconden detrás de la búsqueda del triunfo, provocando con ello no solo el desprestigio 
de otros sino el incremento de la desinformación. En ese contexto, la verificación de hechos y del discurso político se ha hecho más prominente en los últimos años (Nyhan et al., 2019) y ha puesto en alerta no solo a los políticos y a sus equipos de campaña, sino también a los periodistas y medios de comunicación frente a la reproducción de declaraciones sin verificación.

Sin duda las TIC han trastocado el modo de hacer campaña política, trasladando al escenario digital las diferentes estrategias para promocionar a un candidato y a su proyecto político; sin embargo, la expansión acelerada de las fake news abren un nuevo frente de acción en los procesos electorales, en los que, además de idear la forma de llevar a un candidato al triunfo, hay que confrontar los rumores y mentiras que se generan en torno a su figura y proyecto. A esto se suma un conjunto de nuevos hábitos en el uso y consumo de la información en medio de una época en la cual más del $50 \%$ de la población mundial tiene acceso a Internet y un $49 \%$ es usuario activo de las redes sociales (We are Social \& Hootsuite, 2020), espacios clave para la difusión de la desinformación.

Con la atención apuntando hacia las redes sociales es preciso considerar algunos fenómenos que se producen, por la naturaleza que tienen y las posibilidades que ofrecen. Una de ellas es el prosumismo tecnológico (Toffler, 1979), actividades que antes eran mediadas por terceros, y hoy las realiza el usuario sin censura ni control de ningún tipo. Este cambio en el ecosistema digital ha fomentado una multiplicidad de fuentes informativas y datos que circulan en la Red, que están supeditados a las motivaciones y objetivos particulares de los usuarios que los crean y difunden, los cuales pueden ir desde la necesidad de informar algo de interés, pasando por el deseo de hacer una broma, hasta distorsionar una realidad de forma intencional. Ese ha sido el contexto que ha posibilitado la diseminación de fake news, esa información manipulada con fines específicos, generalmente políticos, con el objetivo de cambiar una imagen sobre algo o alguien, y que son presentadas de modo que impacten y provoquen una idea de realidad a través de las plataformas digitales, desde las cuales los usuarios coadyuvan a su difusión (Tandoc et al., 2018). No es lo mismo que los errores en la información por falta de pericia en la investigación de un hecho, sino que encierran una intencionalidad e imitan los contenidos de los medios masivos (Tandoc et al., 2018), su formato y estilo, pero carecen de normas editoriales, fuentes e investigación.

Los efectos negativos provocados por la diseminación de fake news han puesto de manifiesto diferentes iniciativas para su control y erradicación, algunas tienen que ver con la creación de leyes y sanciones destinadas a prohi- 
bir y castigar a quienes difundan o produzcan información falsa, varios países de Europa ya han puesto en marcha leyes en contra de las fake news las cuales no están exentas de críticas y del inminente peligro de la censura al estar en manos de los gobiernos el poder de decidir qué es mentira y qué no en la Red (Valarezo-Cambizaca \& Rodríguez-Hidalgo, 2019).

Otras iniciativas nacen desde el periodismo, como la profesión que por naturaleza desarrolla procesos de investigación, contraste y verificación de los hechos y que actualmente una de las claves en la lucha contra el fenómeno desinformativo. Pero también está una tercera alternativa, cuya visión es en el largo plazo, se trata de la educación en medios o alfabetización mediática, a través de la cual los ciudadanos desarrollen un pensamiento crítico y reflexivo en el uso y consumo de medios e información (Romero-Rodríguez \& Rodríguez-Hidalgo, 2019).

La presente investigación se enmarca en la segunda alternativa: la verificación de hechos o fact-checking, como parte del poder interpretativo que tiene el periodismo en los procesos de actuación política (Luengo \& GarcíaMarín, 2020) y como un elemento necesario que permita al ciudadano tomar decisiones basadas en la información de calidad.

Para sustentar este se revisaron investigaciones anteriores sobre fact-checking y procesos lectorales, las cuales han expuesto el incremento de prácticas de verificación informativa liderada especialmente por periodistas, y sugieren que estas pueden reducir las percepciones erróneas en el discurso político, aunque ello no se refleje en la evaluación de los candidatos y los resultados electorales (Nyhan et al., 2019). Otros como Hill (2017) sostienen que los hechos políticos, ciertos o falsos, generan prejuicios en los votantes solo si estos se identifican con sus creencias preexistentes, y adicionan una gran cantidad de detalles para asemejarse al producto de una investigación (Chan et al., 2017), mientras que para Guess et al. (2020) los niveles de afectación por rumores y mentiras están directamente relacionados con los medios de comunicación que los usuarios consumen de manera permanente en Internet.

A pesar de que los rumores, ataques y campañas de desprestigio nunca han sido ajenas a los procesos electorales, el efecto global que le da Internet a la información, potencia el efecto nocivo de la desinformación en cuanto a la forma como los ciudadanos miran el mundo, conciben lo que es real, correcto y deseable, considerando que es a partir de la información que las personas toman decisiones, como quiénes serán las autoridades que manejen los destinos de sus comunidades. 
No es menos cierto que los procesos de verificación informativa generan cierta resistencia entre los equipos de campaña, de ahí que quienes confían menos en los verificadores, son más vulnerables a sufrir la influencia de la desinformación (Lyons et al., 2020). A esto se suma la necesidad del acceso a datos abiertos que permitan a los periodistas comparar afirmaciones, cifras e información de diverso tipo, con fuentes confiables de datos, incluso hoy países como Estados Unidos, Reino Unido y otros, trabajan en los procesos de verificación con el uso de sistemas de inteligencia artificial que agilita esa búsqueda y contraste de datos.

El debate final entre los candidatos que aspiraban a la presidencia de Estados Unidos en 2020 fue el espacio idóneo para evidenciar el incremento en la difusión de mentiras en las redes sociales, las cuales tuvieron como principal componente el propio discurso de los candidatos presidenciales. Cientos de periodistas de medios de comunicación internacionales como The New York Times, la BBC de Londres, CNN, y de organizaciones especializadas en fact-checking como Politifact, Maldita.es, y otras fueron protagonistas la noche del 22 de octubre cuando desmintieron las aseveraciones de los candidatos durante sus intervenciones.

Este artículo recoge las experiencias electorales de Ecuador y España a través de la verificación informativa en las elecciones celebradas durante en 2019 en cada país con la finalidad de evidenciar la relevancia de la verificación informativa como una línea del periodismo que contrarresta los efectos negativos de la desinformación.

El documento presenta, en primera instancia, una revisión sistemática de la literatura, con el propósito de conceptualizar las fake news y el fact-checking como elementos claves de la investigación al tiempo de indicar cuál es el estado actual del tema verificación informativa en el contexto políticoelectoral; posteriormente, se explica la metodología, objetivos y preguntas de investigación, muestra del estudio e instrumentos utilizados; en seguida se realiza el análisis de los resultados, seguido de la discusión y conclusiones, donde se observan los aportes más significativos de la investigación.

\section{Estado de la cuestión}

\section{Elecciones y desinformación}

Tradicionalmente, los procesos electorales han sido el terreno fértil para la difusión de fake news debido a que las redes sociales constituyen herra- 
mientas cruciales en el desarrollo de campañas políticas debido a las posibilidades de cercanía que construye de cara al usuario (Rodríguez-Hidalgo \& Silva, 2018). La desacreditación de candidatos, proyectos políticos, declaraciones son comunes en todo proceso electoral (Rincón, 2011), y ha sido principalmente la oposición política la encargada de desmentir a un candidato político, y a esta se suma el periodismo con el fact-checking.

La diseminación de fake news provoca efectos adversos en la sociedad y debilita la democracia, no por nada la Unesco hace un llamado de alerta hacia la información falsa, y al desarrollo de un pensamiento crítico (Frau-Meigs, 2017) frente al que constituye tanto un peligro tecnológico como geopolítico de relevancia, cuyos efectos ya se han visto reflejados en eventos electorales recientes como el Brexit, referéndum de la Unión Europea, y las elecciones de Estados Unidos (Elías, 2018). Ambos hechos, sucedidos en el 2016, coadyuvaron a la institucionalización de lo que se conoce como la 'era de la posverdad', y donde se evidenció la búsqueda de intereses particulares a través de cuestionables estrategias de convencimiento masivo que promueven el debilitamiento de la democracia (Ayres-Pinto \& Moraes, 2020).

Adicional a ello, es en el segundo de estos contextos donde toma forma el término fake news cuando, Donald Trump, recientemente electo como presidente de Estados Unidos, utilizó la combinación fake news para desacreditar a la prensa, y posteriormente a la oposición política y a todos quienes que cuestionaran su administración (Baum-Baicker, 2020). Ello obedece a una estrategia política que busca opacar toda información negativa y modificar las percepciones de los ciudadanos, en cuyo proceso el uso de los medios y de la información es fundamental en la forma de legitimar a un líder político (Lischka, 2019).

Las mentiras forman parte habitual del juego político, pero a decir de Cotrino- Zárate (2019), la clave para manipular a la opinión pública está en quién las dice, aunque también se destacan factores como: la forma cómo lo dice, los medios que utiliza y los efectos que provoca en los destinatarios.

En el caso de España, el gobierno tiene su atención focalizada en la difusión fake

news, pero también la academia y algunas organizaciones como la Comisión Europea, que en no pocas ocasiones ha manifestado su preocupación por la alta exposición a la desinformación, junto a la necesidad de medidas de control y de concienciación de las personas (Comisión Europea, 2019).

Durante el referéndum por la independencia de Cataluña circuló un sinnúmero de redes sociales fotografías sacadas de contexto sobre violencia, 
corrupción y otras (Serrat, 2019). Una de las más populares fue la de un grupo de policías agrediendo a ciudadanos, y aunque horas después se comprobó que se trataba de una foto de una manifestación del año 2013, el desmentido no fue tan popular como el rumor.

Lo propio sucede en Ecuador, donde diariamente se difunden fotografías con supuestas declaraciones del presidente Lenín Moreno, que se desmientan posteriormente. Pero al igual que en España, y en otros contextos, los desmentidos no se hacen virales, los rumores sí, ello obedece al gran componente emocional que tienen las fake news, sumado a las construcciones narrativas: memes, fotos y videos alterados, inventados o sacados de contexto.

El alto componente emocional en las fake news se ve fortalecido por la escasa exposición a información de calidad por parte de los usuarios, y por la actividad netamente emocional de los usuarios en redes sociales, no por nada Facebook ha colocado como principal forma de interacción con las publicaciones de usuarios un conjunto de seis emociones con sus respectivos emojis: me gusta, me entristece, me asombra, me divierte, me encanta y me enoja (Valarezo-Cambizaca \& Rodríguez-Hidalgo, 2019).

De ahí que siempre que las fake news son tomadas como ciertas, es porque detrás hay una ideología que la quiere confirmar. A esto se suman campañas orquestadas con ayuda de trolls y bots para fortalecer estrategias cuyo fin es la desinformación haciendo eco de mensajes que se requiere que lleguen a toda la población.

\section{El periodismo frente a las fake news}

Las redes sociales han acaparado notablemente la atención de los usuarios de Internet. Dentro de ellas desarrollan actividades de socialización y entretenimiento (Rivera- Rogel et al., 2018), pero también de información. Ante esto es preciso señalar que las redes sociales no son redes de información, son redes para el intercambio, la socialización, la emoción y por supuesto para la difusión de contenidos, pero no garantizan la veracidad de los contenidos que se difunden a través de ellas, por ello no se puede exigir a las redes sociales la misma responsabilidad que se exige tanto a los periodistas como a los medios de comunicación respecto al manejo informativo (Rodríguez-Hidalgo et al., 2020). 
A pesar de ello, las multinacionales Facebook, Google y WhatsApp han incursionado en acciones de cara a frenar la difusión de información falsa, lo que se ha visto motivado principalmente por el exceso de rumores en redes sociales durante la emergencia provocada por la COVID-19, y que llegó a ser calificado con el término 'infodemia' (Organización Mundial de la Salud, 2020). Ante ello, el periodismo tiene un papel fundamental que es el de brindar respuestas y orientar a la opinión pública con información de calidad buscar y garantizar la verdad como valor máximo (Craft \& Davis, 2013).

Es por esa razón que en los últimos tres años han surgido una serie de organismos dedicados a la verificación de los hechos que se difunden como verdades en la Red (Álvarez \& López, 2016), todos ellos liderados por periodistas. La Silla Vacía en Colombia, Ojo Público en Perú, Ecuador Chequea en Ecuador, Chequeado.com en Argentina, Maldito Bulo en España; FactCheck.org y Politifact.com en los Estados Unidos y otras, son algunas de las organizaciones dedicadas al fact-checking en el continente.

Durante el 2020 la emergencia sanitaria global impulsó la creación de la Coronavirus Fact-Checking Alliance, un proyecto del Poynter Institute y de la Red Internacional de Fact-Checking (IFCN por sus siglas en inglés), y en la cual se encuentran suscritas alredededor de ochenta organizaciones de más de cuarenta países del mundo, para desmentir los rumores y mentiras que se difundieron en la Red sobre la pandemia.

Contrarrestar los rumores y mentiras no solo posibilita un mejor conocimiento del entorno, sino darle al ciudadano la posibilidad de tomar decisiones mejor informadas, considerando que los medios constituyen las ventanas a través de las cuales las personas conocen el mundo y acceden a información sobre temas, contextos y hechos que no experimentan de forma directa (Kovach \& Rosenstiel, 2001). Para este fin, la calidad de la información es clave, y la verificación constituye una de las competencias indiscutibles de los periodistas, que permiten que esa información no se convierta en el eco de lo que señalan las fuentes; $y$, en un contexto electoral, los candidatos que aspiran a una dignidad de elección popular.

Para Álvarez y López (2016) la mentira es parte del juego político, por ello se sobredimensionan los resultados de las encuestas, por ejemplo, de ahí que la comprobación de datos, discursos y hechos tiene además una dimensión educativa para el elector, pero también para la clase política que requiere siempre de cifras, y para el periodismo que utiliza estas cifras como parte de las informaciones que publica. Las audiencias van a los medios en busca 
de aclarar sus dudas y a obtener información que les permita dilucidar sobre la mejor opción de voto, por ello la práctica de la verificación de hechos como una disciplina del periodismo cambiaría notablemente las reglas del contexto electoral, fomentando una mayor responsabilidad de los candidatos a la hora de hacer afirmaciones frente a la opinión pública.

La presente investigación se enfoca en las actividades de verificación que realizaron Maldito Bulo en España y Ecuador Chequea en Ecuador, en los comicios electorales de marzo y abril de 2019, con el propósito de identificar los principales desmentidos de ambos proyectos y destacar la labor de la verificación como esa actividad trascendental del periodismo que garantiza la calidad de la información que circula en un contexto electoral.

\section{Material y métodos}

Se realizó el estudio de las métricas de las cuentas de Twitter de las dos organizaciones dedicadas a verificar la información en cada país: Ecuador Chequea en Ecuador y Maldito Bulo en España, con el objetivo de estudiar los rumores que desmienten con mayor frecuencia y los temas y personajes mayormente sometidos a verificación.

El estudio de las métricas posibilita entender cómo producen las transformaciones sociales gracias a la influencia de las redes sociales, que se encargan de configurar algunas tendencias y patrones sociológicos con la emotividad como su principal componente (Magallón, 2019).

Las preguntas de investigación que orientan esta investigación son: ¿Qué tipo de rumores se desmienten con mayor frecuencia? ¿Quiénes son personajes y temas presentes en los contenidos desmentidos? ¿Cuál es la metodología que aplican los proyectos de fact-checking de Ecuador y España?

Para responder estas preguntas se propone una investigación de tipo cuantitativo. Esta forma de estudio permite aplicar instrumentos metodológicos que se pueden utilizar tanto sobre contenido como continente (Bardin, 2002) al tiempo de ser un tipo de procedimiento replicable que permite inferir los resultados, así como realizar una descripción cuantitativa de una realidad en este caso a través de estadística descriptiva (Berelson, 1952; Krippendorff, 2004).

En ese contexto, se recabó un total de 135 tuits de Ecuador Chequea y 172 de Maldito Bulo publicados entre el 1 de febrero y el 30 abril de 2019, 
que contempla el tiempo antes, durante y después de las elecciones en Ecuador y España. En ese periodo de tiempo ambos países celebraron comicios para elegir alcaldes, concejales, prefectos y miembros de juntas parroquiales, en el caso de Ecuador; y, diputados y senadores en el caso de España.

A partir de los datos recuperados se propone una clasificación de los rumores en siete categorías en las que se agrupan los contenidos numéricamente mayores y que son comunes entre ambos países: declaraciones falsas atribuidas a candidatos propuestas de campaña de los candidatos, publicación de encuestas falsas, presentación de hechos ocurridos en otros países como locales, que fueron parte de un estudio previo realizado por Magallón (2019), a los que se suman otros tres: migración, por ser un tema común entre ambos países, anuncio de fraude electoral y resultados electorales falsos.

Del mismo modo a partir de la revisión de los proyectos Ecuador Chequea y Maldito Bulo se realiza el análisis de contenido de sus metodologías con el fin de identificar prácticas comunes e innovadoras en el proceso de verificación informativa: categorías de las verificaciones, selección de verificables, proceso de verificación y difusión de los verificados.

\section{Análisis y discusión de los resultados}

La política y la migración, son los temas sobre los cuales se difundieron más rumores, y por lo tanto sobre los cuales se hizo el mayor número de desmentidos (Figura 1). En el contexto político, por obvias razones el proceso electoral fue el principal en las noticias e informaciones difundidas en medios y redes sociales: el desarrollo de la campaña, declaraciones, discursos, confrontaciones, preparación de las elecciones, entre otros.

Por otra parte, el tema migratorio, que ocupa el segundo lugar, refleja que existe un marcado interés en ambos países debido a la presencia numerosa de migrantes de diferentes nacionalidades, razón por la cual es un tema presente en el discurso político electoral. También se encontró otro tipo de temáticas relacionadas a encuestas, propuestas de campaña, fraude electoral, resultados finales falsos, en menos cantidad.

En Ecuador Chequea del $45 \%$ de los posts enmarcados en el tema político se observan publicaciones relacionadas a declaraciones falsas atribuidas a candidatos, anuncio de resultados electorales falsos, incoherencias en planes de campaña difundidos en redes, financiamiento de campañas electorales, en- 
tre otros. El $32 \%$ de los posts se enfocan en temas migratorios que aluden de forma especial a los ciudadanos venezolanos quienes en el último año han llegado masivamente al país producto de la crisis política que atraviesa.

Figura 1

\section{Temática de los posteos desmentidos}

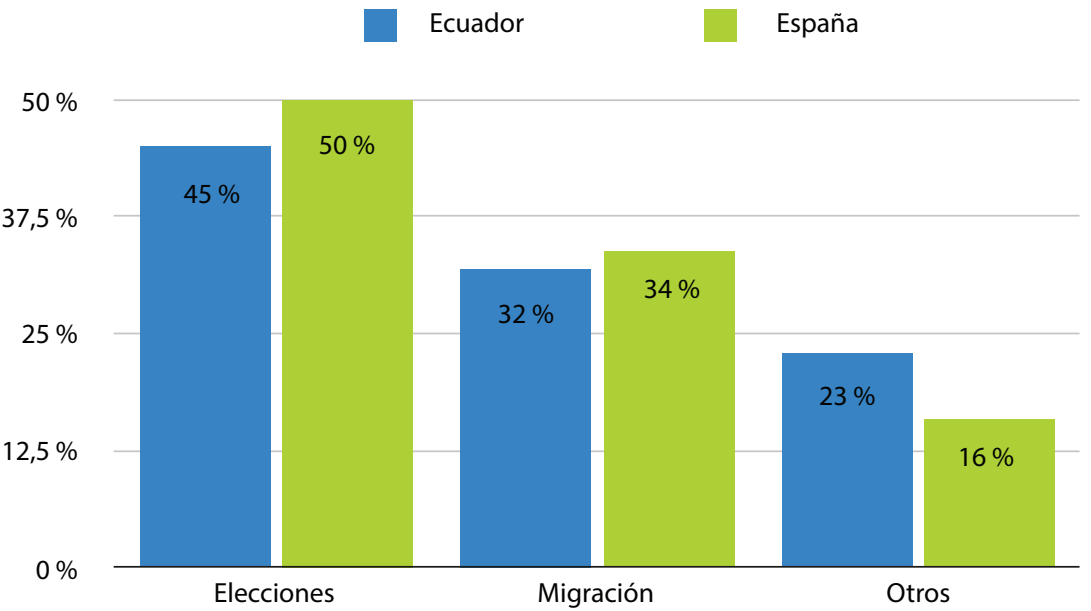

Fuente: Ecuador Chequea, n.d.-a; Maldito Bulo, n.d.

Elaboración propia.

Los resultados de España no se diferencian demasiado del caso ecuatoriano. Un 50\% de contenidos corresponden a temas políticos especialmente relacionados a los candidatos y partidos que participaron en las elecciones de abril de 2019, papeletas impresas erróneamente, presunción de fraude electoral, declaraciones falsas de candidatos, creación de cuentas parodia de candidatos, protestas de estudiantes y ciudadanos en general, entre otros. Los rumores y mentiras se presentaron principalmente en forma de supuestas declaraciones con fotografías y videos manipulados y sacados de contexto que atribuían a un candidato declaraciones no dichas, o pronunciadas en un contexto diferente.

Un 34\% alude al tema migratorio con notas como el cobro de 1200 euros al mes por parte de inmigrantes ilegales, modificación de la ley de seguridad social, censura de Podemos hacia procesiones ofensiva para ciudadanos, la 
matanza de corderos por parte de musulmanes. Del total de publicaciones enmarcadas en el ámbito político se realizó la clasificación de los rumores, los cuales se aprecian en la Figura 2.

\section{Figura 2 \\ Tipos de rumores difundidos en las elecciones}

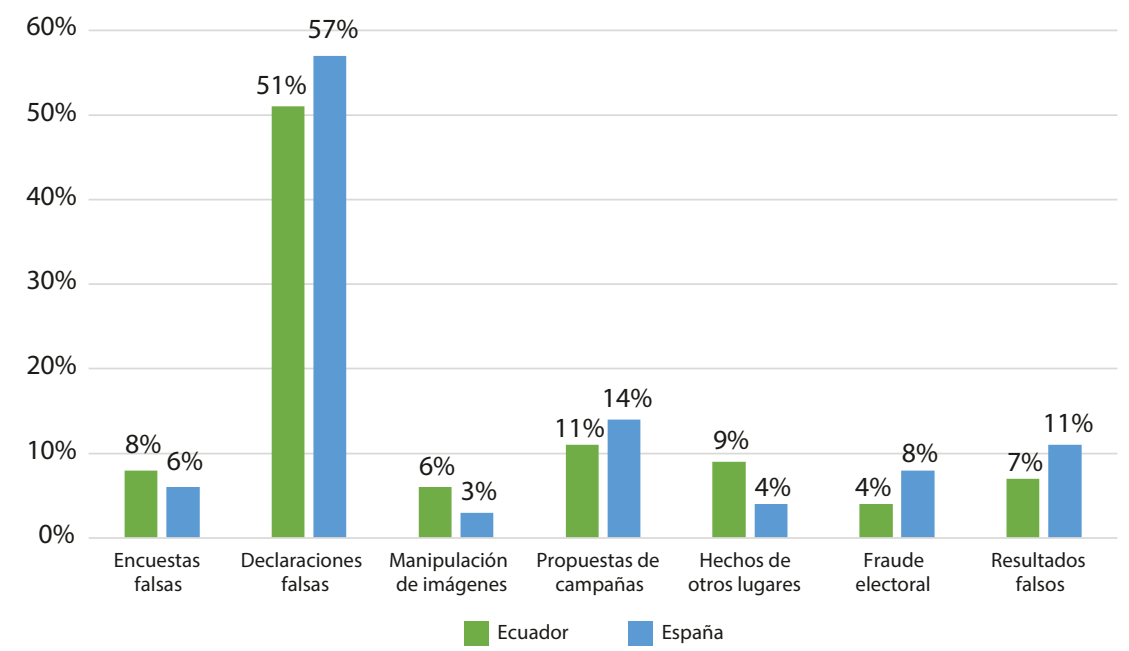

Fuente: Ecuador Chequea, n.d.-a.; Maldito Bulo, n.d.

Elaboración propia.

Las declaraciones falsas se enmarcaron en a la coyuntura de cada país. Por ejemplo, en Ecuador, al candidato Jorge Yunda, se le atribuyó la siguiente declaración en una entrevista televisada: "el verdadero problema de la ciudad es el mal genio, y eso sabemos combatirlo" (Ecuador Chequea, 2019), con ella se intentó posicionar una imagen poco será de quien pretendía ser el alcalde de la capital del país.

Otro caso es el supuesto tuit de Pablo Iglesias, candidato de Podemos diciendo "ojalá una bomba ahora en Colón ¿Dónde están los terroristas cuando se les necesita?" en relación con una manifestación por la unidad de España realizada en febrero de 2019. 
En ambos casos los desmentidos se realizaron con la búsqueda de las declaraciones originales: el video, en el caso de Yunda, y el tuit en el caso de Iglesias, ninguno existía.

En segundo lugar, Ecuador Chequea, acude a la fuente, Yunda, para cuestionar sobre las declaraciones y estas fueron negadas, sumado a ello se publica el video original, al que se atribuye la falsa declaración y no se menciona nada parecido a la falsa publicación.

Otro tema recurrente es el relacionado a la propuesta de campaña de los candidatos y el anuncio de resultados electorales falsos. Ejemplo de lo antes mencionado se observan en la Figura 3 que recoge dos tuits: el primero de Maldito Bulo sobre alertas de fraudes electorales en España, y el segundo, el supuesto anuncio de la candidata Gabriela Rivadeneira de la Lista 5 como el posicionamiento de este partido como la primera fuerza política de Ecuador, ambos desmentidos.

\section{Figura 3}

\section{Otros temas desmentidos}

MB

MALDITO BULO

@malditobulo

Alertas de fraudes electorales del 28A de los que no hay pruebas.

\#VotaSinBulos

\#EleccionesGenerales28A

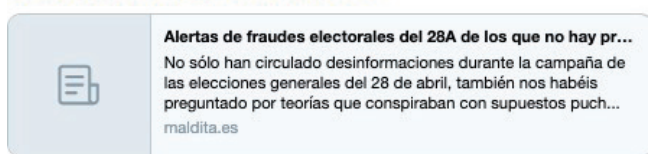

$1: 08-28$ abr. 2019

37 Retweets $\mathbf{3 4}$ Me gust

2000องคค
CCuAdon Ecuador Chequea

Aunque el movimiento utilizado por el expresidente Correa para postular sus candidatos logró las prefectura de Pichincha y Manabí, la lista 5 no alcanzó ninguna de las 49 alcaldías que buscó... \#NoComasCuento ow.ly/RSPw30ojrtz

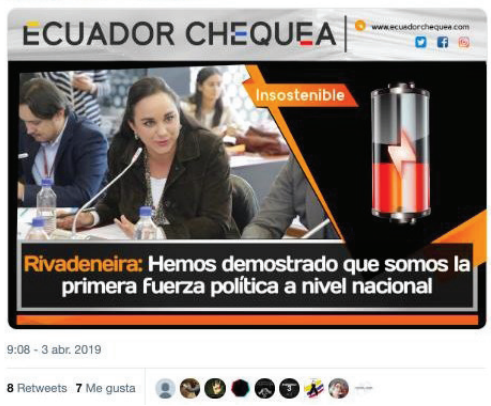

Fuente: Ecuador Chequea, n.d.-a; Maldito Bulo, n.d.

Elaboración propia.

En cuanto al nivel de interacción de los usuarios con los temas con los desmentidos por las cuentas de fact-checking de Ecuador y España se obser- 
va una gran diferencia entre ambos. La cuenta de Ecuador Chequea tiene un nivel de interacción muy bajo de sus usuarios en comparación con Maldito Bulo en donde se observa una gran cantidad de retuits y favoritos en las publicaciones. Ecuador Chequea obtiene un 10\% de la interacción de los usuarios con sus desmentidos, mientras que Maldito Bulo tiene al menos un 70\% en promedio. Del mismo modo se evidenció que las reacciones frente a los desmentidos que realizan ambas organizaciones no tienen la misma viralidad que los rumores. En la Figura 4 se observa la relación de la popularidad entre fake news y desmentidos.

\section{Figura 4}

\section{Relación de popularidad entre desmentidos y rumores}

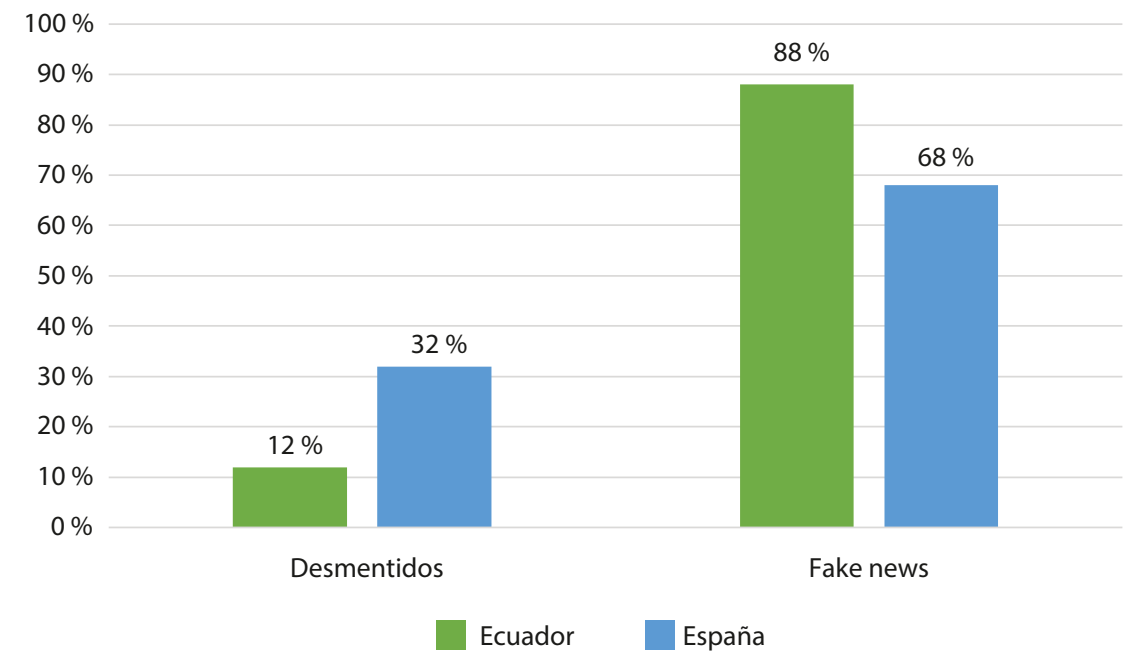

Fuente: Ecuador Chequea, n.d.-a; Maldito Bulo, n.d.

Elaboración propia.

Para ampliar la información de la imagen anterior, la Tabla 1 expone las cinco publicaciones sometidas a verificación y que tienen el mayor número de interacciones en cada país, ahí se aprecia la diferencia entre ambos países, en cuanto a la interacción con información verificada. 


\section{Tabla 1}

Principales rumores desmentidos en las campañas electorales

\begin{tabular}{|c|c|c|c|c|c|}
\hline @EcuadorChequea & Fav & RT & @MalditoBulo & Fav & RT \\
\hline $\begin{array}{l}\text { @ MashiRafael dice que le están } \\
\text { robando los vocales del Consejo de } \\
\text { Participación. "Ladrón que roba a } \\
\text { ladrón". }\end{array}$ & 47 & 41 & $\begin{array}{l}\text { No. Este vídeo no es de la } \\
\text { manifestación de hoy en } \\
\text { Colón. Aparece el edificio } \\
\text { Barclays derruido el año } \\
\text { pasado. No está la publicidad } \\
\text { de Netflix que sí está hoy en } \\
\text { Colón. }\end{array}$ & $5.6 \mathrm{~K}$ & $6.5 \mathrm{~K}$ \\
\hline $\begin{array}{l}\text { @ PaolaVintimilla está en lo correcto. } \\
\text { Los datos del Municipio de Quito } \\
\text { reflejan, a febrero de 2019, un total de } \\
19.253 \text { empleados. La cifra incluye } \\
\text { funcionarios de la administración } \\
\text { municipal y de las empresas públicas } \\
\text { municipales... \#NoComasCuento }\end{array}$ & 29 & 18 & $\begin{array}{l}\text { No, no hay pruebas de que } \\
\text { vecinos de Madrid hayan } \\
\text { quitado cintas contra orugas } \\
\text { pensando que eran lazos } \\
\text { amarillos }\end{array}$ & 909 & $1.1 \mathrm{~K}$ \\
\hline $\begin{array}{l}\text { La información es falsa. Tras un } \\
\text { monitoreo a la entrevista de La } \\
\text { Posta Ec, no se encontró la frase } \\
\text { que se atribuye a @LoroHomero... } \\
\text { \#NoComasCuento y revisa https://t. } \\
\text { co/TPPdSrWD1Q https://t. } \\
\text { co/8SNq16suXj }\end{array}$ & 13 & 11 & $\begin{array}{l}\text { No. Pablo Iglesias ni ha } \\
\text { publicado ni ha borrado } \\
\text { un tuit diciendo "ojalá una } \\
\text { bomba ahora en Colón". Es } \\
\text { un tuit falso, no hay ninguna } \\
\text { prueba de su publicación. } \\
\text { Solo una captura (la misma) } \\
\text { que se está viralizando como } \\
\text { real siendo falsa. https:// } \\
\text { twitter.com/malditobulo/ } \\
\text { status/1094900978485211136 }\end{array}$ & 726 & 973 \\
\hline $\begin{array}{l}\text { Aunque el movimiento utilizado por } \\
\text { el expresidente Correa para postular } \\
\text { sus candidatos logró las prefecturas } \\
\text { de Pichincha y Manabí, la lista } 5 \text { no } \\
\text { alcanzó ninguna de las } 49 \text { alcaldías } \\
\text { que buscó... \#NoComasCuento } \\
\text { https://t.co/86H66sMk3Y https://t.co/ } \\
\text { wU2fukhxFa }\end{array}$ & 7 & 8 & $\begin{array}{l}\text { No, la supuesta encuesta } \\
\text { del Diario de Andorra que } \\
\text { da } 82 \text { escaños a Vox no } \\
\text { existe: es un montaje. https:// } \\
\text { twitter.com/malditobulo/ } \\
\text { status/1122160203099721728 }\end{array}$ & 532 & 647 \\
\hline
\end{tabular}

Fuente: Ecuador Chequea, n.d.-a; Maldito Bulo, n.d.

Elaboración propia.

Los desmentidos sobre estos cinco posts se desarrollan a través de tres prácticas principales identificadas en ambos casos (Figura 5). La más común es la búsqueda de información en medios de comunicación, principalmente en la prensa; en segundo lugar, en el caso de declaraciones de un candidato, buscan en sus redes sociales evidencias que lo que se supone que dice. 
Finalmente, en ambos casos acuden a fuentes oficiales, para verificar información sobre gastos, estadísticas y otros datos, desarrollando el ejercicio periodístico de contraste informativo, así como entrevistas con las fuentes involucradas en el rumor, lo cual permite además de verificar un rumor, informar con base en información producida por el propio medio.

En algunos casos se observa incluso, el uso de fuentes expertas que coadyuven a la explicación de un tema y con ello fomentar la comprensión de un tema, además de esclarecer un rumor.

Se destaca el trabajo de Ecuador Chequea en revisión de documentos de planes de gobierno y comparación entre ellos, en este proceso se observa un trabajo con base en información oficial con el cual se elaboró infografías destinadas a informar al electorado, más que a desmentir rumores.

\section{Figura 5 \\ Técnicas para desmentir fake news}

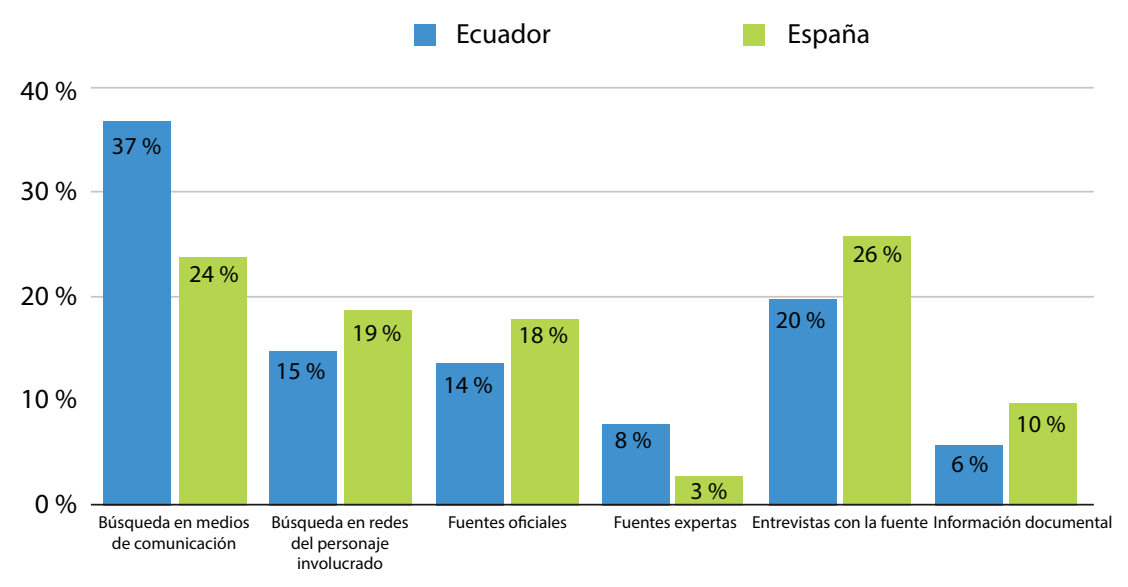

Fuente: Ecuador Chequea, n.d.-a; Maldito Bulo, n.d.

Elaboración propia.

En lo que respecta a Maldito Bulo, se explica que si la verificación no puede realizarse a través de las estrategias antes mencionadas se acude a otras alternativas que tienen que ver con niveles más específicos de acceso a la información. 
En ocasiones, si incluso después de llevar a cabo la debida diligencia no se puede demostrar la falsedad del mensaje, pero se cree que se puede acceder a información adicional haciendo uso de la ley de transparencia, se solicita la información adicional para dar la visión más completa del hecho. (Maldita.es, n.d.)

El desmentido o verificación por lo general contiene la mayor cantidad de detalles posibles, junto a fotografías y en algunos casos videos que ayudan a sostener la verdad sobre el rumor que se intenta derribar. Adicional a ello ambas iniciativas se aseguran de etiquetar cada rumor que ha llegado a sus manos con diferentes categorías, algunas de las cuales se asemejan entre ellas como se observa a continuación:

Tabla 2

Categorías de verificación

\begin{tabular}{|l|l|}
\hline \multicolumn{1}{|c|}{ Ecuador Chequea } & \multicolumn{1}{c|}{ Maldito Bulo } \\
\hline Cierto & \\
\hline Sí, pero & Verdadero, pero \\
\hline Insostenible & \\
\hline Falso & Falso \\
\hline & Falso, pero \\
\hline
\end{tabular}

Fuente: Ecuador Chequea, n.d.-b; Maldita.es, n.d.

Elaboración propia.

En el caso de Maldito Bulo cuando un rumor sometido a verificación se comprueba como verdadero, este no se publica en redes, por ello no incluyen esa categoría; sin embargo, según se expresa en la Metodología de Maldito Dato, 2019, se puede utilizar esta calificación cuando se trata de una confrontación entre dos partes que requiere de una aclaración hacia el público.

\section{Conclusiones}

El flujo de ingentes cantidades de información, de muchos emisores a muchos receptores, incrementa la necesidad de proveedores de información calificados, que se dediquen a la verificación de hechos. 
La tarea de verificar los hechos que realizan Ecuador Chequea y Maldito Bulo permite dimensionar el aporte del periodismo, como aquella profesión que tiene una responsabilidad social con la calidad de la información y de la misma manera el papel del periodista, como el profesional que desarrolla el compromiso de trabajar con la información y el conjunto de actividades enmarcadas en la recopilación de datos, verificación, de declaraciones, documentos, y otros que le permitan elaborar los contenidos que transmite al público a través de distintos soportes de forma oportuna y con calidad (Muratova et al., 2019).

A esto se suma que las dos iniciativas de fact-checking estudiadas se encuentran conformadas por grupos de periodistas que se encargan tanto de la valoración de contenidos como de la verificación informativa. Lo cual llama a reconsiderar al periodismo, tan venido a menos, como esa profesión que tiene como obligación máxima la verdad; no se habla de la verdad en un sentido filosófico, sino en el sentido periodístico, el cual contempla el ejercicio de la verificación de hechos como uno de sus principios básicos, y al mismo tiempo la práctica que posibilita la creación de contenidos informativos basados en hechos.

La desinformación, y de modo especial las fake news, con sus altos contenidos emocionales, calan más profundamente en las personas que una información verificada lo cual es preocupante, pero al mismo tiempo refleja la necesidad de desarrollar competencias digitales que le permitan al usuario discriminar contenidos en la red y utilizar la información de forma crítica.

No es menos cierto que los diferentes fenómenos desinformativos también aprovechan la ausencia de datos e informaciones que ayuden a las personas a conocer un tema y no les permitan creer con facilidad un rumor que se intenta colar como verdad; claro está, que eso también depende de los niveles de exposición informativa de las personas y los temas a los cuales eligen exponerse.

Como era de esperarse, en un contexto electoral el proceso en sí mismo constituyó el tema principal de las mentiras difundidas en la Red, y a pesar de que el $45 \%$ de desmentidos de Ecuador y $50 \%$ de desmentidos de España se enmarcan en ese tema, no se puede obviar a la migración como un tema sensible en ambos países, razón por la cual los desmentidos tienen un nivel de prevalencia mayor al $30 \%$ en relación con otros como resultados de encuestas y sospechas de fraude electoral.

La verificación de hechos constituye una clave de cara a garantizar la verdad en la información que se divulga, al tiempo de evitar que los medios 
se limiten a ser cajas de resonancia del discurso político, y que en su lugar identifiquen y aclaren informaciones inexactas antes de su difusión. Esto deja entrever el valor de la verificación y la investigación, otros estudios podrían indagar sobre la media en que los periodistas desarrollan ambas en el contexto desinformativo actual.

La contribución más evidente de este trabajo es la visión del periodismo en la función de garantizar la verdad, en un entorno donde existe muchos emisores dirigiéndose a muchos receptores al mismo tiempo, y donde es preciso desacreditar la mentira y fortalecer la verificación como una disciplina.

\section{Bibliografía}

Álvarez, L., \& López, X. (2016). El Fact checking como herramienta de combate contra el sensacionalismo. XIV Congreso Asociación Historiadores de la Comunicación, 87-102. https://bit.ly/3iAEfot

Ayres-Pinto, D.J., \& Moraes, I. (2020). Digital media as a tool of manipulation in democratic electoral processes: An analysis of the brexit case. $R e$ vista de Estudios Sociales, 2020(74), 71-82. https://doi.org/10.7440/ res74.2020.06

Bardin, L. (2002). Análisis de contenido. Akal. S.A. https://bit.ly/3iEdSOr

Baum-Baicker, C. (2020). Not Fake News: Toxic Consequences of the Trump Stress Effect. Journal of Humanistic Psychology, 60(6), 730-746. https://doi. org/10.1177/0022167820911757

Berelson, B. (1952). Content analysis in communication research. Free Press.

Chan, M.S., Jones, C., Jamieson, K., \& Albarracín, D. (2017). Debunking: A Meta-Analysis of the Psychological Efficacy of Messages Countering Misinformation. Psychological Science, 28(11), 1531-1546. https://doi. org/10.1177/0956797617714579

Comisión Europea (2019). Unión Europea vs. Desinformación / España. https://bit. ly/3bXh8TM

Consejo Nacional Electoral (2019). Consejo Nacional Electoral-Construyendo Democracia-Ecuador. Voto de Ecuatorianos en el Exterior. https://bit. ly/3ocW1Oq

Cotrino-Zárate, A. C. (2019). La mentira en política. Entre la manipulación de los hechos y la pregunta por quién habla ahí. Universitas Philosophica, 36(72), 71-95. https://doi.org/10.11144/Javeriana.uph36-72.mhpq 
Craft, S., \& Davis, C. (2013). Principles of American Journalism: An Introduction. Routhledge. https://bit.ly/3sJfyeh

Ecuador Chequea (n.d.-a). @Ecuadorchequea. https://twitter.com/ECUADORCHEQUEA (n.d.-b). Metodología-Ecuador Chequea. https://bit.ly/2LfOtym (2019). \#FALSETA Yunda afirmó que los quiteños quieren un alcalde que los haga reír. Verificaciones. https://bit.ly/3bXhoSK

Elías, C. (2018). Fakenews, poder y periodismo en la era de la posverdad y "hechos alternativos." Ámbitos: Revista Internacional de Comunicación, 40, 1-6. https://bit.ly/3qMAxL4

Frau-Meigs, D. (2017). Contra la información falsa, espíritu crítico. Organización de las Naciones Unidas para la Educación, la Ciencia y la Cultura. https://bit.ly/3iChUGS

Guess, A., Nyhab, B., \& Reifler, J. (2020). Exposure to untrustworthy websites in the 2016 US election. Nature Human Behaviour. https://doi.org/10.1038/ s41562-020-0833-x

Hill, S. (2017). Learning Together Slowly: Bayesian Learning about Political Facts. Journal of Politics, 79(4), 1403-1418. https://doi.org/10.1086/692739

Kovach, B., \& Rosenstiel, T. (2001). The Elements of Journalism: What Newspeople Should Know and the Public. Crown. https://bit.ly/3qW2IHV

Krippendorff, K. (2004). Content Analysis: An Introduction to Its Methodology. SAGE Publications Ltd. https://bit.ly/2NkL2qE

Lischka, J. A. (2019). A Badge of Honor?: How The New York Times discredits President Trump's fake news accusations. Journalism Studies, 20(2), 287-304. https://doi.org/10.1080/1461670X.2017.1375385

Luengo, M., \& García-Marín, D. (2020). The performance of truth: politicians, factchecking journalism, and the struggle to tackle COVID-19 misinformation. American Journal of Cultural Sociology. https://doi.org/10.1057/ s41290-020-00115-w

Lyons, B., Mérola, V., \& Reifler, J. (2020). How Politics Shape Views Toward Fact-Checking: Evidence from Six European Countries. International Journal of Press-Politics, 25(3), 469-492. https://doi.org/https://doi. org/10.1177/1940161220921732

Magallón, R. (2019). Verificado México 2018. Disinformation and fact-checking on electoral campaign. Revista de Comunicacion, 18(1), 234-258. https:// doi.org/10.26441/RC18.1-2019-A12 
Maldita.es. (n.d.). Metodología de Maldito Dato. Metodología de Maldito Dato. https://bit.ly/3a1zjFo

Maldito Bulo. (n.d.). @malditobulo.https://twitter.com/malditobulo

Muratova, N., Grizzle, A., \& Mirzakhedova, D. (2019). Media and Information Literacy in Journalism: A handbook for journalists and journalism educators. Unesco. https://bit.ly/2Y56kux

Nyhan, B., Porter, E., Reifler, J., \& Wood, T.J. (2019). Taking Fact-checks Literally But Not Seriously? The Effects of Journalistic Fact-checking on Factual Beliefs and Candidate Favorability. Political Behavior, 42(3), 939-960. https://doi.org/10.1007/s11109-019-09528-x

Organización Mundial de la Salud (2020). Primera Conferencia de la OMS sobre Infodemiología. https://bit.ly/3pdRr55

Rincón, O. (2011). Mucho ciberactivismo... pocos votos. Antans Mockus y el Partido Verde colombiano. Nueva Sociedad, 235, 74-89. https://bit.ly/3papFGG

Rivera-Rogel, D., Velásquez-Benavides, A., \& Rodríguez-Hidalgo, C. (2018). Socialización, ocio y entretenimiento, los principales usos del smartphone en nativos digitales de Ecuador. En R. Cruz-Díaz, F. Caro-González, \&

A. Ramírez-García (Eds.), Uso del teléfono móvil, juvebntud y familia (pp. 129-143). https://bit.ly/3p9G667

Rodríguez-Hidalgo, C., Mier-Sanmartín, C., \& Coronel-Salas, G. (2020). Fake news y política: Nuevos desafíos para las campañas electorales. RISTI Revista Ibérica de Sistemas e Tecnologias de Informacao, 35, 351-362. https://bit.ly/3sO06xb

Rodríguez-Hidalgo, C., \& Silva, J. (2018). Política 2.0: Facebook como herramienta de campaña. Estudio de caso elecciones generales Ecuador 2017. En M.J. Pérez- Serrano, G. Alcolea-Díaz, \& A. Nogales-Bocio (Eds.), Poder y medios en las sociedades del siglo XXI (pp. 63-85). Egregius. https:// bit.ly/3sNtHa8

Romero-Rodríguez, L. M., \& Rodríguez-Hidalgo, C. (2019). Desinformación y posverdad en los medios digitales: del astroturfing al click-baiting. [Disinformation and post-truth in digital media: from astroturfing to clickbaiting]. En La comunicación en el escenario digital. Actualidad, retos y prospectivas (pp. 379-401). Pearson. https://bit.ly/3p8zW68

Serrat, J. (2019). Voter in the streets on October 1, 2017 in Catalonia: an example of covert fake news. Trípodos, 44, 153-168. https://bit.ly/3oDUqCJ

Tandoc, E., Ling, R., Westlund, O., Duffy, A., Goh, D., \& Zheng Wei, L. (2018). Audiences' acts of authentication in the age of fake news: A conceptual 
framework. New Media \& Society, 20(8), 2745-2763. https://doi.org/ https://doi.org/10.1177/1461444817731756

Toffler, A. (1979). La tercera ola. Terra, 241.

Valarezo-Cambizaca, L.M., \& Rodríguez-Hidalgo, C. (2019). Innovation in journalism as an antidote to fake news. RISTI - Revista Ibérica de Sistemas e Tecnologias de Informacao, E20, 24-35. https://bit.ly/3p9scAN

We are Social \& Hootsuite (2020). Digital 2020. Global Digital Overview. https:// bit.ly/3p5K $5 \mathrm{k} 2$

Fecha de envío: 2020/10/24; Fecha de aceptación: 2021/01/19;

Fecha de publicación: 2021/03/01 